\title{
NAHRUNGSERGÄNZUNG \\ Fischöl gegen den Muskelabbau bei Krebspatienten
}

- Patienten, die sich einer Chemotherapie unterziehen müssen, verlieren oft an Gewicht und Muskelmasse. Wie eine aktuelle Studie in Cancer online zeigt, könnte eine Nahrungsergänzung mit Fischöl dies verhindern. Zwei Patientengruppen mit neu diagnostiziertem nicht kleinzelligem Lungenkarzinom unterzogen sich der Standardtherapie. Die Probanden einer Gruppe erhielten über zehn Wochen zusätzlich Fischöl in Form von 2,2 Gramm Eicosapentaensäure pro Tag. Bei allen Patienten wurden Gewicht, Muskelmasse und Fettanteil kontrolliert.

Die meisten Patienten ohne Fischölsupplementation verloren durchschnittlich $2,3 \mathrm{~kg}$ während der Chemotherapie. Nur 29\% der Teilnehmer dieser Kontrollgruppe nahmen nicht ab. Dagegen konnten 69\% der Patienten, die zusätzlich Fischöl erhielten, ihr Körpergewicht konstant halten oder gewannen sogar Muskelmasse hinzu. Zudem zeigte sich ein dosisabhängiger Effekt: Je höher die Eikosapentaensäure-Konzentration im Blut war, desto mehr Muskeln bauten die Patienten auf. Hinsichtlich der Gesamtmasse an Fettgewebe gab es zwischen den Gruppen dagegen keinen Unterschied.

Nach Ansicht der Autoren könnten Patienten während einer Standardkrebstherapie von 2,2 g Fischöl täglich profitie- ren. Die Autoren hoffen, nun endlich eine Möglichkeit gefunden zu haben, die krebsbedingte Mangelernährung in den Griff zu bekommen. Denn die Supplementation eignet sich auch für Patienten mit anderen Krebsarten oder chronischen Krankheiten, die mit Mangelernährung assoziiert sind.

Murphy RA, et al. Cancer. Article first published online: 28 FEB 2011. DOI: 10.1002/cncr.25709

\section{ERSCHRECKENDE VORBILDER}

\section{Ritzende Teenies: der Renner auf YouTube}

- Wer bei YouTube das Stichwort "Selbstverletzung" eingibt, wird erschreckend leicht fündig: Jugendliche, die sich die Handgelenke ritzen, Verbrennungen zufügen, die Haut mit spitzen Gegenständen durchbohren, all das teilweise in Großaufnahme, unkommentiert und frei zugänglich. Dass die makabren Filmchen mit hoher Frequenz geklickt werden, zeigt eine kanadische Studie, die die meistgesehenen Videos zu den Stichworten "Self-injury" und "Self-harm" sowie die Bewertungen der Online-User analysierten. Die Top 100 wurden demnach über 2 Millionen mal angesehen; $80 \%$ waren ohne Altersbeschränkung zugänglich, $58 \%$ enthielten keinen Hinweis auf eine möglicherweise jugendgefährdende Wirkung. Über 12 000-mal wurden die Sequenzen als Favoriten ausgewählt, also von den Betrachtern als positiv bewertet.

Den Forschern zufolge besteht die Gefahr solcher Videos darin, dass sie zum Nachmachen anregen können. Frühere Studien haben gezeigt, dass selbstverletzendes Verhalten ohne Selbstmordabsicht bei Kindern, Jugendlichen und jungen Erwachsenen in bis zu 21\% auftritt. Die Betroffenen

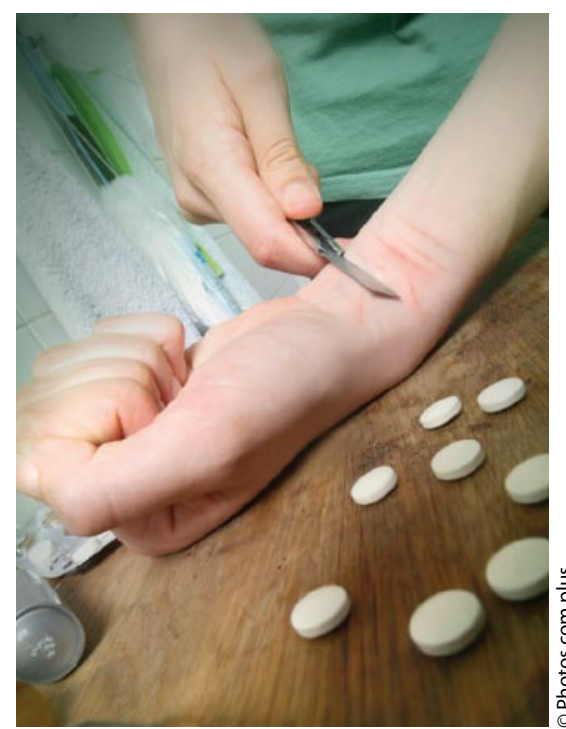

Selbstverletzungsvideos regen zum Nachahmen an.

zeigten vermehrt zwischenmenschliche Probleme, psychiatrische Symptome und wären auch stärker suizidgefährdet.

EO -

Lewis SP, et al. Pediatrics online, 21. Februar 2011; doi: 10.1542/peds.2010-2317

\section{GROSSSTADTRISIKO}

\section{Luftverschmutzung: Haupttrigger für Infarkt}

- Dass die Luftverschmutzung Einfluss auf Herz-Kreislauf-Erkrankungen hat, ist lange bekannt. Doch nun haben belgische und Schweizer Wissenschaftler verschiedene Trigger für nicht tödliche Herzinfarkte vom Kokainkonsum über den Kaffeeverbrauch bis hin zum Sex miteinander verglichen.

Dabei stellte sich heraus: Die Luftverschmutzung ist einer der wichtigsten Auslöser für einen Infarkt. Ihr Anteil ist in etwa gleichzusetzen mit den Auswirkungen von akuter körperlicher Anstrengung oder den Folgen von Alkohol und Koffein.

Somit könnte auch die steigende Zahl koronarer Herzerkrankungen in den Ballungsgebieten von Entwicklungsländern die zunehmende Luftverschmutzung widerspiegeln.

ST .

Nawrot TS, et al. The Lancet. Published online February 24,2011 